\title{
Autofluorescence spectroscopy for in vivo diagnosis of DMBA-induced hamster buccal pouch pre-cancers and cancers
}

\author{
Chih-Yu Wang ${ }^{1}$ \\ Tsuimin Tsai $^{2}$ \\ Hui-Chun Chen ${ }^{2}$ \\ Shu-Chen Chang ${ }^{3}$ \\ Chin-Tin Chen ${ }^{4, *}$ \\ Chun-Pin Chiang ${ }^{5,6}$, \\ ${ }^{1}$ Department of Biomedical Engineering, I-Shou \\ University, \\ ${ }^{2}$ Graduate Institute of Biomedical Materials, \\ Taipei Medical University, \\ ${ }^{3}$ Department of Food Sanitation, Tajen Institute \\ of Technology, \\ ${ }^{4}$ Center for Optoelectronic Biomedicine, \\ ${ }^{5}$ School of Dentistry, College of Medicine, \\ National Taiwan University, and \\ ${ }^{6}$ Department of Dentistry, National Taiwan \\ University Hospital, Taipei, Taiwan \\ *These two authors have equally contributed in \\ this paper.
}

\begin{abstract}
Background: Our previous ex vivo study has shown that autofluorescence spectroscopy at 330-nm excitation can discriminate specimens of normal buccal pouch mucosa (normal), epithelial hyperkeratosis (hyperkeratosis), epithelial dysplasia (dysplasia), and squamous cell carcinoma (SCC) taken from DMBA-treated hamsters by using the method of partial least-squares discriminant analysis (PLSDA).

Methods: This study used a fiber optics-based fluorescence spectroscopy system to measure the autofluorescence spectra of 23 normal, 14 hyperkeratosis, 28 dysplasia, and 10 SCC samples in vivo. PLSDA with cross-validation was used to analyze the autofluorescence spectral data of all samples.

Results: We found that at 330-nm excitation, the autofluorescence spectra of all samples had two main peaks: one at $380 \mathrm{~nm}$ and the other at $460 \mathrm{~nm}$. The hyperkeratosis samples had a higher 380-nm emission peak (EP) and a lower 460-nm EP than normal samples. On the contrary, the dysplasia samples had a lower 380-nm EP and a higher 460-nm EP than normal samples. Furthermore, the SCC samples had a much lower 380-nm EP and a much higher 460-nm EP than all other samples. To quantify the spectral changes during the progression of oral carcinogenesis, ratios of the area under the spectrum of $380 \pm 15 \mathrm{~nm}$ to that under the spectrum of $460 \pm 15 \mathrm{~nm}$ (denoted as $A_{380 \pm 15 \mathrm{~nm} /}$ $\left.A_{460 \pm 15 \mathrm{~nm}}\right)$ for all samples were calculated. The mean ratio values of $A_{380 \pm 15 \mathrm{~nm}} / A_{460 \pm 15 \mathrm{~nm}}$ decreased gradually from hyperkeratosis to normal, to dysplasia, and to SCC samples. Significant differences in this mean ratio were found between any two groups of normal, hyperkeratosis, dysplasia, and SCC samples. By choosing proper thresholds, PLSDA with cross-validation could provide an accurate identification rate of $86 \%$ for hyperkeratosis, of $87 \%$ for normal, and of $89 \%$ for dysplasia samples. In addition, by choosing a proper threshold, we could separate benign (normal and hyperkeratosis) from dysplasia or SCC tissues with a sensitivity of $92 \%$ and a specificity of 95\%.

Conclusion: Our results indicate that the autofluorescence spectroscopy technique is a useful diagnostic tool for in vivo diagnosis of oral pre-cancers and cancers in DMBA-induced hamster buccal pouch carcinogenesis model.
\end{abstract}

Key words: autofluorescence spectroscopy; in vivo measurement; oral carcinogenesis

J Oral Pathol Med 2003: 32: 18-24 
Tobacco, areca quid and alcohol are recognized to be associated with several kinds of head and neck cancers $(1,2)$. Because of the increased consumption of these stimulators, head and neck cancers have gradually become important problems of health care in many countries (3). In Taiwan, there are 2 million people who habitually chew areca quids (4); approximately $80 \%$ of all oral cancer deaths are associated with this habit (5). The survival rate of oral cancer increases dramatically if the oral cancer can be found and treated in its early stage. Therefore, to develop a diagnostic tool that can detect oral pre-cancers and early oral cancers would be important for the high-risk patients.

Autofluorescence spectroscopy has been shown to be a promising technique for diagnosis of cancers arising from a variety of organs (6-19). Studies on using autofluorescence spectroscopy to detect head and neck or oral cancers gradually increase in recent years, indicating the importance of using this technique for diagnosis of head and neck or oral cancers (10-19). For easy control of experimental conditions, some researchers used DMBA-induced hamster buccal pouch carcinogenesis model to test the feasibility of using autofluorescence spectroscopy to diagnose oral pre-malignant and malignant lesions (14-19). In an ex vivo study, Balasubramanian et al. (14) analyzed the fluorescence spectra of a few extracted fluorophores from hamster buccal pouches and found that excitation at 405 and $420 \mathrm{~nm}$ gave significant differences in fluorescence spectra among normal, premalignant, and malignant tissues. Wang et al. (15) have developed a probability-based algorithm for oral cancer identification, achieving up to $81 \%$ of accuracy rate for specimens taken from DMBA-treated hamster buccal pouches in four stages of cancer development. Chen et al. (16) found that under 330-nm excitation wavelength, significant differences in intensity of 380- and 460-nm peaks of autofluorescence spectra are observed exvivo among hamster normal, pre-malignant and malignant buccal pouch tissues. In an in vivo study, Pathak et al. (17) showed that autofluorescence imaging could distinguish abnormal from normal hamster buccal pouch tissues with a sensitivity of $76 \%$ and a specificity of $83 \%$. Using multiple laser wavelengths (337, 380 and $460 \mathrm{~nm}$ ) as excitation light, Coghlan et al. (18) demonstrated that algorithms based on autofluorescence could separate neoplastic from non-neoplastic buccal pouch tissues in a DMBA-induced hamster carcinogenesis model. These results from previous studies indicate the potential of using autofluorescence spectroscopy to detect pre-cancerous and cancerous lesions in human beings and in DMBA-treated hamsters.

Our previous ex vivo study has shown that autofluorescence spectroscopy at 330-nm excitation can discriminate specimens of normal buccal pouch mucosa (normal), epithelial hyperkeratosis (hyperkeratosis), epithelial dysplasia (dysplasia), and squamous cell carcinoma (SCC) taken from DMBA-treated hamsters by using the method of partial least-squares (PLS) discriminant analysis (19). To test whether this diagnostic tool can also be applied to DMBA-treated hamsters in vivo, we employed a fiber optics-based fluorescence spectroscopy system to measure the in vivo autofluorescence spectra of 75 buccal pouch sites including normal mucosa and lesions at different stages of carcinogenesis. PLS discriminant analysis with cross-validation was used to analyze the autofluorescence spectral data of all samples. We found that at 330-nm excitation, each category (normal, hyperkeratosis, dysplasia, or SCC) of samples had its own specific autofluorescence spectral pattern. By choosing proper thresholds, PLS discriminant analysis with cross-validation could provide an accurate identification rate of $86 \%$ for hyperkeratosis, of $87 \%$ for normal, and of $89 \%$ for dysplasia samples. In addition, by choosing a proper threshold, we could separate benign (normal and hyperkeratosis) from pre-malignant (dysplasia) or malignant (SCC) tissues with a sensitivity of $92 \%$ and a specificity of $95 \%$. Our results indicate that the autofluorescence spectroscopy technique is a useful diagnostic tool for in vivo diagnosis of oral pre-cancers and cancers in DMBA-induced hamster buccal pouch carcinogenesis model.

\section{Materials and methods}

\section{Animals}

There were 23 8-week-old male Syrian golden hamsters used in this study. These hamsters were painted with a $0.5 \%$ solution of carcinogen, DMBA, in heavy mineral oil on their left buccal pouches three times a week. The right buccal pouches were left untreated and used as normal controls.

\section{Autofluorescence spectroscopy and tissue processing}

In this study, a total of 75 (52 abnormal and 23 normal) buccal pouch sites were measured to obtain the autofluorescence spectra. A fiber optics-based fluorescence spectrometer (Spex SkinSkan, JY Inc., Edison, NJ, USA) was used for the measurement. A monochromator with a 150-W ozone-free Xenon lamp was used as the excitation light source. The excitation light was guided to illuminate samples by one arm of a Y-type quartz fiber bundle, and the emission fluorescence was collected by another arm of the fiber bundle, guided to another motor-controlled 
monochromator. The intensity of the emission light at each wavelength was detected with a photomultiplier tube (Hamamatsu K.K., Hamamatsu, Japan). The excitation wavelength was chosen to be $330 \mathrm{~nm}$ because the excitation wavelength around $330 \mathrm{~nm}$ has been shown to be one of the optimal wavelengths for neoplasia tissue discrimination in our previous ex vivo studies $(16,19)$. The resulting emission spectra were recorded from 340 to $601 \mathrm{~nm}$, in 3 -nm increments.

The spectral measurement was performed with the hamsters being under anesthesia. The buccal pouches were pulled out and fixed flatly on a suberic plate with metal tweezers. The optical fiber tip was gently touched on the measuring sites and autofluorescence spectra were measured. To precisely localize the measuring sites, the periphery of the measuring area was tattooed with India ink. For avoiding the possible photobleaching effect, each sample was measured only twice. It was found that the intensity change of two repeated measurements was less than $2 \%$. After each measurement, the optical fiber tip was carefully cleaned to remove the possible remaining drug on the tip. The hamsters were sacrificed immediately after measurement. The buccal pouch tissues of the measured sites were excised, fixed in $10 \%$ neutral formalin for $6 \mathrm{~h}$, embedded, sectioned, and stained with hematoxylin and eosin. An oral pathologist who was blinded to the fluorescence results performed all histopathological assessments in another laboratory. The histopathological results were recorded and used for further analysis.

For each hamster, there might exist more than one grade of carcinogenesis in its buccal pouch simultaneously. For example, one hamster with a lesion of SCC probably also had dysplasia or hyperkeratosis on the mucosa around the SCC lesion. Therefore, for each left buccal pouch, we measured the autofluorescence spectra of more than one site. All the measured tissues were excised for histopathological examination. The samples were pooled together and used for statistical analysis to check the accuracy of spectroscopic classification.

\section{Sample classification}

Table 1 shows the histological assessment results and numbers of the samples. A total of 75 samples were divided into four categories: normal $(n=23)$, hyperkeratosis $(n=14)$, dysplasia $(n=28)$, and SCC $(n=10)$. To carry out the partial least-squares (PLS) discriminant analysis, the former three categories were coded with two-digit dummy variables (see Table 1). The SCC samples were not adopted for our statistical algorithm because the SCC samples had the distinct fluorescence pattern that was apparently different from and could be easily separated from
Table 1. Histological assessment results and numbers of samples

\begin{tabular}{llll}
\hline Category & Number & $\begin{array}{l}\text { Coded dummy } \\
\text { variable }\end{array}$ & \\
\hline Normal buccal pouch mucosa & 23 & 0 & 0 \\
Epithelial hyperkeratosis & 14 & 0 & 1 \\
Epithelial dysplasia & 28 & 1 & 0 \\
Squamous cell carcinoma & 10 & - & - \\
\hline
\end{tabular}

The coded dummy variable is also shown in the table. The squamous cell carcinoma samples were not included in our statistical algorithm, thus were not coded with dummy variables

those of normal, hyperkeratosis, and dysplasia samples. The absolute intensity of the spectra might vary with some factors, including the excitation power, the angle between tissues and optical fiber tip, and the diffuse reflectance of tissues (20). To avoid the possible uncertainty, all spectra were normalized by dividing the intensity at each wavelength by the integrated area under the total spectrum. The normalized spectra had the same area under the curve, with the pattern unchanged. The normalized spectra were then saved for further data analysis.

\section{PLS discriminant analysis with cross-validation technique}

PLS discriminant analysis with cross-validation technique is a multivariate statistical method, which extracts the most important factors that relate to the categories. By calculating the least average error of prediction result, the optimal number of PLS scores can be determined with cross-validation technique (21, 22). After being calculated, the PLS score can be used to make a PLS plot, in which the samples in different categories can be classified. In general, the first several PLS scores would contain most discriminant information, and thus would be most proper for PLS plot. The details for this method were shown in our previous works (19).

\section{Results}

\section{Autofluorescence spectra}

Figure 1 shows the typical autofluorescence spectra of the normal, hyperkeratosis, dysplasia, and SCC samples at 330-nm excitation. All the curves had two main peaks with broad bands: one was around $380 \mathrm{~nm}$ and the other was around $460 \mathrm{~nm}$. Compared to the spectra of normal samples, the spectra pattern started to change at the stage of hyperkeratosis: the intensity of the 380-nm emission peak (EP) increased, while that of the 460-nm EP decreased. When the buccal pouch mucosa became dysplastic, 


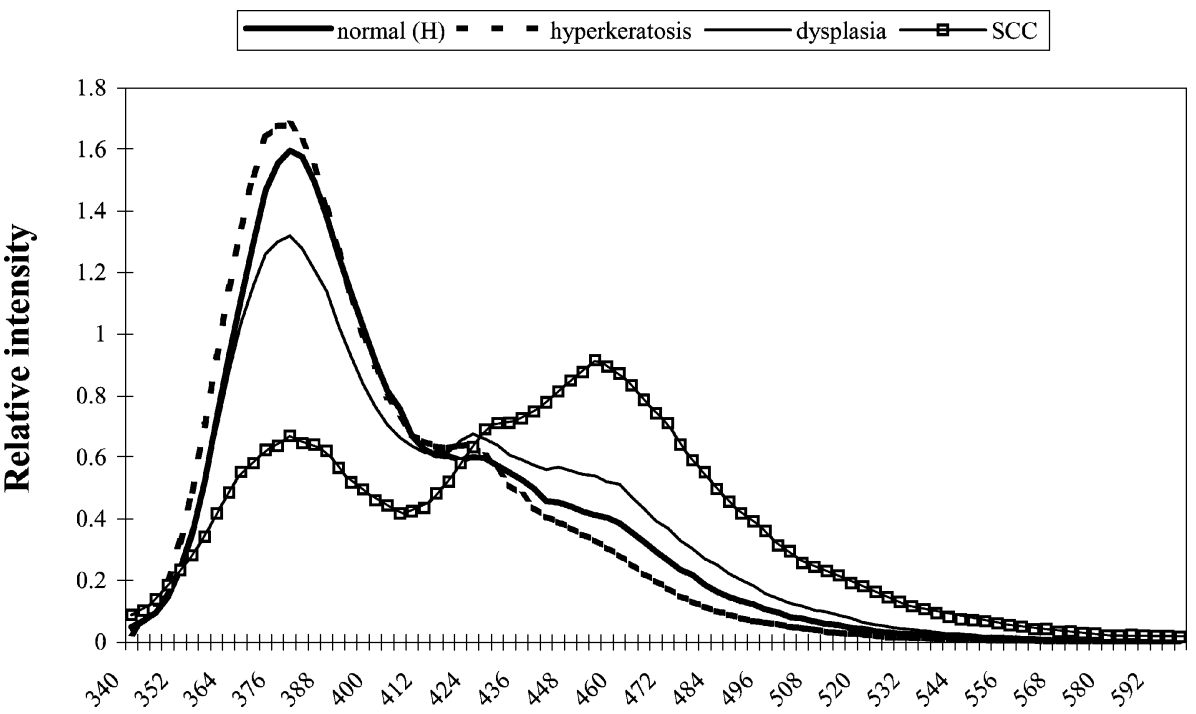

Fig. 1. Typical autofluorescence spectra of normal, hyperkeratosis, dysplasia, and squamous cell carcinoma (SCC) samples.

\section{Emission wavelength (nm)}

the intensity of the 380-nm EP reduced, while that of the 460-nm EP increased. This tendency of alternation became more apparent when the dysplastic lesion transformed into SCC (Fig. 1). These primary patterns of the autofluorescence spectra for the present in vivo study were similar to those observed in our previous ex vivo studies $(16,19)$. Therefore, the analyzing methods developed in our previous exvivo studies might be applicable for the present in vivo measurement data.

Besides the relative changes of the 380 and 460-nm main peaks, the absolute intensities of the fluorescence spectra were also different during the progression of buccal pouch carcinogenesis. In general, the absolute intensities became lower when the buccal pouch mucosa changed from benignity to malignancy.

To simply illustrate how the spectral changes were related to buccal pouch mucosa transformation from benignity to malignancy, ratios of the area under the spectrum of $380 \pm 15 \mathrm{~nm}$ to that under the spectrum of $460 \pm 15 \mathrm{~nm}$ (denoted as $A_{380 \pm 15 \mathrm{~nm}} /$ $A_{460 \pm 15 \mathrm{~nm}}$ ) were calculated. Figure 2 shows the average values

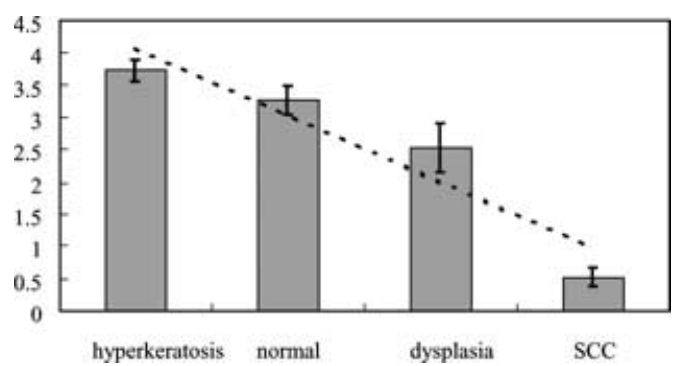

Fig. 2. The average values of the ratio values of $A_{380 \pm 15 \mathrm{~nm}} / A_{460 \pm 15 \mathrm{~nm}}$ for hyperkeratosis, normal, dysplasia, and squamous cell carcinoma (SCC) samples. The error bars for each category and tide line are also shown. of $A_{380 \pm 15 \mathrm{~nm}} / A_{460 \pm 15 \mathrm{~nm}}$ for hyperkeratosis, normal, dysplasia and SCC samples. The mean ratio values decreased gradually from hyperkeratosis to normal, to dysplasia, and to SCC. An ANOVA test showed significant differences in the ratio value among all categories of samples (Table 2). To further identify the relationship between groups, a multiple comparisons method, which is a technique for more than three categories by drawing their $100(1-\alpha) \%$ confidence interval (23), was employed. If the interval (denoted by $[L, U]$ contains zero, the mean values of two categories may be equal under (1- $\alpha)$ significant level. If $L>0$ (or $U<0$ ), the mean difference for two categories may be positive (or negative) under $(1-\alpha)$ significant level. Figure 3 shows that with $\alpha=0.01$, all intervals are positive, indicating that $\mu_{\mathrm{HYP}}>\mu_{\mathrm{NOR}}>$ $\mu_{\mathrm{DYP}}>\mu_{\mathrm{SCC}}$. These results indicate that each category has its own specific ratio of $A_{380 \pm 15 \mathrm{~nm}} / A_{460 \pm 15 \mathrm{~nm}}$ and it is possible to develop a diagnostic algorithm for the hyperkeratosis, dysplasia, and SCC lesions by using the autofluorescence spectroscopy.

\section{PLS discriminant analysis with cross-validation}

To efficiently evaluate the performance of the classification algorithm, the PLS discriminant analysis was carried out with crossvalidation technique. Figure 4 shows the PLS discriminant plot

Table 2. ANOVA test for the ratio values $\left(\mathrm{A}_{380 \pm 15 \mathrm{~nm}} / \mathrm{A}_{460 \pm 15 \mathrm{~nm}}\right)$ of normal, hyperkeratosis, dysplasia, and squamous cell carcinoma samples

\begin{tabular}{llllll}
\hline Variation source & SS & d.f. & MS & $F$ & $P$-value \\
\hline Between groups & 70.21307 & 3 & 23.4 & 294.09 & $5.93 \mathrm{E}-40(P<0.01)$ \\
Within group & 5.65026 & 71 & 0.079581 & & \\
Sum & 75.86333 & 74 & & & \\
\hline
\end{tabular}




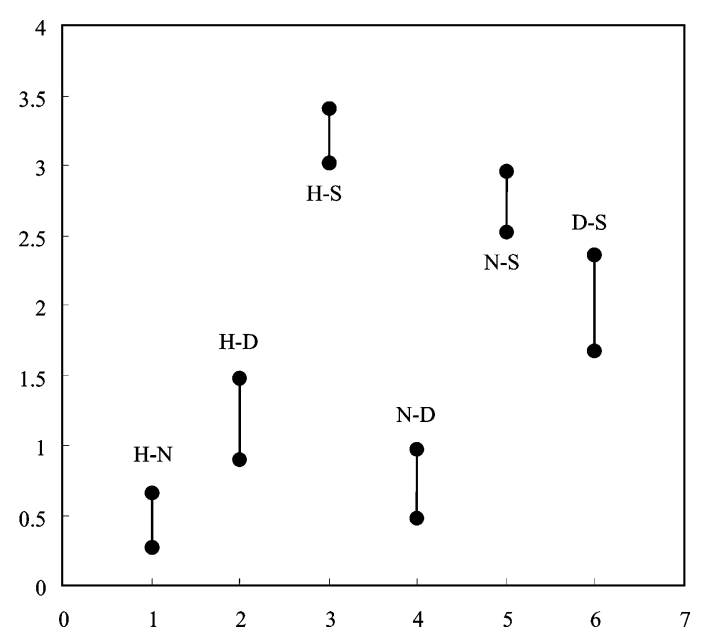

Fig. 3. Results of multiple comparisons method. Notation: H: Hyperkeratosis. N: Normal. D: Dysplasia. S: Squamous cell carcinoma. The notation $\mathrm{H}-\mathrm{N}$ indicated $99 \%$ confidence interval of $\mu_{\mathrm{H}}-\mu_{\mathrm{N}}$, and so on.

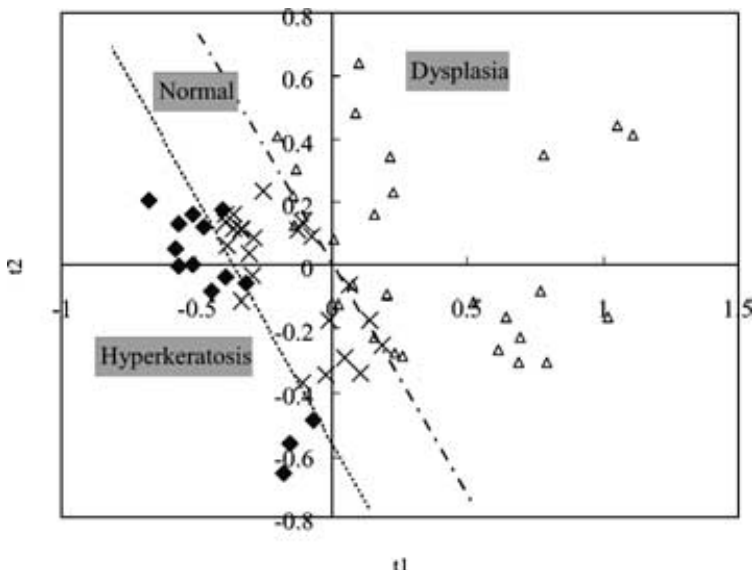

Fig. 4. PLS score plot ( $t_{1}$ vs. $\left.t_{2}\right)$ of normal, hyperkeratosis, and dysplasia samples. The two thresholds separate these samples into three parts. Squamous cell carcinoma samples are not shown on the plot.

( $t_{1}$ vs. $\left.t_{2}\right)$ of normal, hyperkeratosis, and dysplasia samples. The PLS score values of SCC samples were not shown on the plot because they were not included in this calculation. As shown in Fig. 4, the data points of hyperkeratosis, normal, and dysplasia samples were gathered into three groups. By choosing proper thresholds, an accurate identification rate of $86 \%$ for hyperkeratosis, of $87 \%$ for normal, and of $89 \%$ for dysplasia samples was obtained. Sometimes we might just concern about the classification of benign (normal and hyperkeratosis) and pre-malignant (dysplasia) or malignant (SCC) tissues. By choosing a proper threshold, we could separate benign from pre-malignant or malignant tissues with a sensitivity of $92 \%$ and a specificity of $95 \%$.

\section{Discussion}

In this study, we established an identification algorithm for the diagnosis of oral pre-cancers and cancers in a hamster buccal pouch carcinogenesis model using autofluorescence spectroscopy. The normal, hyperkeratosis, and dysplasia samples of buccal pouch tissues could be grouped on the PLS score plot. By applying suitable thresholds, these samples could be identified with accuracy rates of $86-89 \%$. Furthermore, by using the classification algorithm we can discriminate benign from premalignant or malignant tissues with a sensitivity of $92 \%$ and a specificity of $95 \%$. These high accuracy rates and high degree of sensitivity and specificity suggest that the autofluorescence spectroscopy technique with PLS discriminant analysis is a useful diagnostic tool for invivo diagnosis of oral pre-cancers and cancers in DMBA-induced hamster buccal pouch carcinogenesis model.

Although the mechanisms causing the changes of the autofluorescence spectra during the progression of oral cacinogenesis are still not clear, previous studies have demonstrated that the major contributor for the peak around $380-400 \mathrm{~nm}$ is collagen and that for the peak around $440-460 \mathrm{~nm}$ is NADH $(11,24$, 25). Histologically, the hyperkeratosis samples showed a slight increase in keratin and epithelial thickness as well as an infiltrate of chronic inflammatory cells. Cytokines and growth factors produced by inflammatory cells within the connective tissue may promote fibrosis by inducing proliferation of fibroblasts, upregulating collagen synthesis, and downregulating collagenase production (26). Therefore, it was possible that the markedly increased subepithelial collagen content in hyperkeratosis samples raised the 380-nm EP. Furthermore, the effect of increased collagen content on the augmentation of collagenderived emission fluorescence might surpass the effect of slightly thickened epithelium on the reduction of collagenderived emission fluorescence. This caused a higher 380-nm EP of autofluorescence spectra for hyperkeratosis samples than that for normal samples. However, prominent epithelial hyperplasia was often found in dysplasia samples. The thickened and hyperplastic epithelium might reduce the intensity of both excitation energy and collagen-derived emission fluorescence. This resulted in a lower 380-nm EP of autofluorescence spectra for the dysplasia samples than that for normal samples. In SCC samples, cancer cells proliferated outward and inward, which increased the thickness of the cancer tissue. This thickened layer of cancer tissue prevented the excitation light from reaching the underlying collagen layer and filtered out a great part of 
the collagen-derived emission fluorescence. In case of early buccal pouch cancer, part of the excitation light might reach the underlying collagen layer; however, a certain part of the collagen-derived emission fluorescence was filtered out by the slightly thickened cancer tissue. Furthermore, in buccal pouch cancer, only little stromal connective tissue (collagen) was found among cancer nests. This resulted in a much lower 380-nm EP of autofluorescence spectra for SCC samples than those for normal or dysplasia samples.

Free NADH excites at $340 \mathrm{~nm}$ and emits at $450-462 \mathrm{~nm}$ (27). Because $\mathrm{NADH}$ plays key roles in multiple cellular metabolic activities, its cytosol level is parallel to the degree of cellular metabolic activity (27). In buccal pouch dysplasia and cancerous lesions, cell proliferation and mitotic figures are frequently found. Therefore, these dysplasia or SCC lesions are supposed to contain more $\mathrm{NADH}$ than normal buccal pouch mucosa due to increased cellular metabolic activity. The gradual increase in the $\mathrm{NADH}$ content as the normal buccal pouch mucosa transformed into dysplastic and further into cancerous tissues caused a gradual augmentation of the 460-nm EP of the autofluorescence spectra from normal to dysplasia and from dysplasia to SCC samples. In hyperkeratosis samples, no dysplastic cells were found in the epithelium. Therefore, no significant difference in the epithelial $\mathrm{NADH}$ content and in the intensity of the 460-nm EP was found between hyperkeratosis and normal samples. Glasgold et al. (28) reported similar findings of autoflurorescence spectral changes during the process of malignant transformation in an NMBA-induced rat esophageal carcinogenesis model. They found that at 340-nm excitation, a gradual drop of the intensity of the 390-nm EP and a gradual augmentation of the intensity of the 450-nm EP are observed as the hyperplastic tissue transforms into dysplastic and cancerous tissues. Their further experiments proved that the fluorophores responsible for the 390-nm EP exists in the subepithelial connective tissue (collagen) and that (NADH) responsible for the 450-nm EP exists in the epithelium (28).

In summary, the normal, hyperkeratosis, dysplasia, and SCC samples from DMBA-treated hamster buccal pouches had significantly different autofluorescence spectral patterns and mean values of $A_{380 \pm 15 \mathrm{~nm}} / A_{460 \pm 15 \mathrm{~nm}}$. By using PLS discriminant analysis with cross-validation, high accuracy rates for diagnosis of a lesion as hyperkeratosis, normal, and dysplasia could be obtained. By choosing a proper threshold, we could also separate benign from pre-malignant or malignant tissues with a sensitivity of $92 \%$ and a specificity of $95 \%$. These results indicate that the autofluorescence spectroscopy technique has a high potential for in vivo diagnosis of oral pre-cancers and cancers.

\section{References}

1. Parkin DM, Pisani P, Feblay J. Estimates of the worldwide incidence of eighteen major cancers in 1985. Int J Cancer 1993; 54: 594-606.

2. World Health Organization. Control of oral cancer in developing countries - WHO meeting report. World Health Bull 1984; 62: 17-30.

3. Department of Health. The Executive Yuan, ROC Cancer Registry Annual Report, Republic of China 1996.

4. Ko YC, Chiang TA, Chang SJ, Hsieh SF. Prevalence of betel quid chewing habit in Taiwan and related sociodemographic factors. J Oral Pathol Med 1992; 21: 261-4.

5. Kwan HW. A statistical study on oral carcinomas in Taiwan with emphasis on the relationship with betel quid chewing: a preliminary report. J Formos Med Assoc 1976; 75: 497-505.

6. Agrawal A, Utzinger U, Brookner C, Pitris C, Mitchell MF, RichardsKortum R. Fluorescence spectroscopy of the cervix: influence of acetic acid, cervical mucus, and vaginal medications. Lasers Surg Med 1999; 64: 720-35.

7. Ramanujam N, Mitchell MF, Mahadevan-Jansen A, et al. Cervical precancer detection using a multivariate statistical algorithm based on laser-induced fluorescence spectra at multiple excitation wavelengths. Photochem Photobiol 1996; 64: 720-35.

8. Ramanujam N, Mitchell MF, Mahadevan A, et al. In vivo diagnosis of cervical intraepithelial neoplasia using 337-nm-excitation laser-induced fluorescence. Proc Natl Acad Sci USA 1994; 19 10193-7.

9. Wang TD, Crawford JM, Feld MS, Wang Y, Itzkan I, Van Dam J. In vivo identification of colonic dysplasia using fluorescence endoscopic imaging. Gastroint Endosc 1999; 49: 447-55.

10. Schantz SP, Kolli V, Savage $\mathrm{HE}$, et al. In vivo native cellular fluorescence and histological characteristics of head and neck cancer. Clin Cancer Res 1998; 4: 1177-82.

11. Eker C, Ryland R, Svanberg K, Andersson-Engles S. Multivariate analysis of laryngeal fluorescence spectra recorded in vivo. Lasers Surg Med 2001; 28: 259-66.

12. Gillenwater A, Jacob R, Ganeshappa R, et al. Non-invasive diagnosis of oral neoplasia based on fluorescence spectroscopy and native tissue autofluorescence. Arch Otolaryngol Head Neck Surg 1998; 124 1251-8.

13. Ingrams DR, Dhingra JK, Roy K, et al. Autofluorescence characteristics of oral mucosa. Head Neck 1997; 19: 27-32.

14. Balasubramanian S, Elangovan V, Govindasamy S. Fluorescence spectroscopic identification of 7,12-dimethylbenz[a]anthraceneinduced hamster buccal pouch carcinogenesis. Carcinogenesis 1995; 16: $2461-5$

15. Wang CY, Chen CT, Chiang CP, Young ST, Chow SN, Chiang HK. A probability-based multivariate statistical algorithm for autofluorescence spectroscopic identification of oral carcinogenesis. Photochem Photobiol 1999; 69: 471-7.

16. Chen CT, Chiang HK, Chow SN, et al. Autofluorescence in normal and malignant human oral tissues and in DMBA-induced hamster buccal pouch carcinogenesis. J Oral Pathol Med 1998; 27: 470-4.

17. Pathak I, Davis NL, Hsiang YN, Quenville NF, Palcic B. Detection of squamous neoplasia by fluorescence imaging comparing profimer 
sodium fluorescence to tissue autofluorescence in the hamster cheek-pouch model. Am J Surg 1995; 170: 423-6.

18. Coghlan L, Utzinger U, Richards-Kortum R, et al. Fluorescence spectroscopy of epithelial tissue throughout the dysplasia-carcinoma sequency in an animal model: spectroscopic changes precede morphologic changes. Lasers Surg Med 2001; 29: 1-10.

19. Wang C-Y, Chen C-T, Chiang C-P, Young ST, Chow SN, Chiang HK. Partial least-squares discriminant analysis on autofluorescence spectra of oral carcinogenesis. Appl Spectrosc 1998; 52: 1190-6.

20. Qu JY, Hua J. Calibrated fluorescence imaging of tissue in vivo. Appl Opt 2001; 78: 4040-2.

21. Martens H, Naes T. Multivariate calibration. Chichester: Wiley Inc., 1989.

22. Sjostrom M, Wold S, Soderstrom B. PLS discriminant plots. In: Gelsema, ES, Kanal, LN., eds. Pattern Recognition in Practice II. Amsterdam: Elsevier Science Publishers, 1986: 461-70.

23. Hwang CY. Multivariate analysis: an introduction. Taipei: Institute of Chinese Economy and Business, 1995: 20-3.

24. Alfano RR, Das BB. Light sheds light on malignant tumors from benign tissues and tumors. Bull NY Acad Med 1991; 67: 143-50.
25. Bottiroli G, Groce AC, Locatelli D, et al. Natural fluorescence of normal and neoplastic human colon: a comprehensive 'ex vivo' study. Lasers Surg Med 1995; 16: 48-60.

26. Haque MF, Harris M, Meghji S, Barrett AW. Immunolocalization of cytokines and growth factors in oral submucous fibrosis. Cytokine 1998; 10: 713-9.

27. Salarma G, Lombardi R, Elson J. Maps of optical action potential $\mathrm{NADH}$ fluorescence in intact working hearts. Am J Physiol 1987; 252: 384-94.

28. Glasgold R, Glasgold M, Savage H, Pinto J, Alfano R, Schantz S. Tissue autofluorescence as an intermediate endpoint in NMBAinduced esophageal carcinogenesis. Cancer Lett 1994; 82: 33-41.

\section{Acknowledgements}

This study was supported by a research grant of NSC89-2736-L-002-006 and NSC90-2736-L-214-004 from the National Science Council, Taipei, Taiwan. 